\title{
The effect of a non-intensive community-based lifestyle intervention on the prevalence of Metabolic Syndrome. The DEPLAN study in Greece
}

\author{
Konstantinos Makrilakis, ${ }^{1}$ Sofia Grammatikou, ${ }^{1}$ Stavros Liatis, ${ }^{1}$ Meropi Kontogianni, ${ }^{2}$ \\ Despoina Perrea, ${ }^{3}$ Charilaos Dimosthenopoulos, ${ }^{1}$ Kalliopi-Anna Poulia, ${ }^{1}$ \\ Nicholas Katsilambros ${ }^{1}$
}

${ }^{1}$ First Department of Propaedeutic Medicine, Athens University Medical School, Laiko General Hospital, ${ }^{2}$ Department of Nutrition \& Dietetics, Harokopio University, ${ }^{3}$ Laboratory for Experimental Surgery and Surgical Research "Christeas Hall", University of Athens Medical School, Athens, Greece

\begin{abstract}
OBJECTIVE: The aim of the present study was to evaluate the effectiveness of a non-intensive, community-based, lifestyle intervention program on the prevalence of metabolic syndrome (MS), in individuals at high risk for development of type 2 diabetes (T2D). DESIGN: In accordance with the FINDRISC score, 191 high-risk persons for T2D, 56.3 \pm 10.8 years old, participated in a one-year lifestyle intervention program consisting of six bi-monthly sessions with a dietician. MS prevalence was assessed at baseline and one year later. RESULTS: The intervention was completed by 125 participants. They lost on average $1.0 \pm 4.8 \mathrm{~kg}(p=0.025)(\operatorname{mean} \pm \mathrm{SD})$ and registered favourable dietary changes. The baseline prevalence of MS was similar among age groups and genders and decreased after one year (from $63.4 \pm 48.4 \%$ to $54.8 \pm 50.0 \%, p<0.001$ ). In a multiple logistic regression model, younger age $(p=0.009)$, male gender $(p=0.004)$, improvement of the dietary score after one year $(p=0.022)$, a lower FINDRISC score $(p=0.033)$, a lower triglyceride level $(p=0.010)$ and a higher baseline HDL-C level $(p=0.003)$ were significantly and independently associated with improvement in MS status. CONCLUSIONS: A non-intensive lifestyle intervention program to prevent T2D is effective in decreasing the prevalence of MS in individuals at high risk for T2D development, possibly conferring multiple cardiovascular health benefits.
\end{abstract}

KEY WORDS: Lifestyle intervention, Metabolic syndrome, Type 2 diabetes prevention

Address for correspondence:

Konstantinos Makrilakis, MD, MPH, PhD, First Department of Propaedeutic Medicine, University of Athens Medical School, Laiko General Hospital, 17 Ag. Thoma Str., 11527 Athens, Greece, Tel.: +30-210-7456261,

Fax: +30-210-7791839, E-mail: kmakrila@med.uoa.gr

Received 12-01-12, Revised 30-02-12, Accepted 15-03-12

\section{INTRODUCTION}

Metabolic syndrome (MS) is a cluster of associated conditions (mainly obesity, dyslipidemia, hypertension and hyperglycemia $)^{1}$ associated with increased risk of cardiovascular disease $(\mathrm{CVD})^{2}$ and type 2 diabetes mellitus (T2D). ${ }^{3}$ There are several defini- 
tions for the syndrome, leading to some difficulty in comparing data from different studies. ${ }^{4-7}$ The most widely accepted criteria are those of the National Cholesterol Education Program - Adult Treatment Panel III (NCEP-ATP III), ${ }^{6}$ slightly revised in 2005 by the American Heart Association/National Heart, Lung and Blood Institute (AHA/NHLBI) ${ }^{8}$ which are basically in agreement with the recently harmonized criteria of the International Diabetes Federation and other scientific Organizations. ${ }^{9}$

Since obesity and physical inactivity contribute to the increased risk of developing $\mathrm{MS},{ }^{10}$ it is logical to conclude that the incorporation of a prudent diet aiming at normal weight and regular physical activity in the daily routine will prevent the development of the syndrome ${ }^{11,12}$ and reduce its impact on persons already affected by it. ${ }^{13}$

The prevalence of MS varies in different studies, ${ }^{14-16}$ but according to the latest U.S. National Health and Nutrition Examination Survey (1999-2002), it is on average around $34.5 \%$ in the general U.S. population, ${ }^{16}$ the number increasing significantly with age (up to around $42 \%$ at age $>70$ years).${ }^{14}$ Since insulin resistance is considered a key pathophysiological feature of the syndrome, ${ }^{4}$ people with pre-diabetes (impaired fasting glucose [IFG] and/or impaired glucose tolerance [IGT]) should be expected to have a high prevalence. Indeed, in the U.S. Diabetes Prevention Program (DPP), MS (using ATP-III criteria) was present in 53\% of the participants (all with IGT) at baseline (and as much as $69 \%$, when a fasting plasma glucose [FPG] cut-off $>100 \mathrm{mg} / \mathrm{dl}$ [ $5.6 \mathrm{mmol} / \mathrm{L}$ ] was used). It was reduced by $41 \%$ in the lifestyle group compared with the placebo group after the three-year intervention. ${ }^{13}$

The prevalence of MS in Greece has been reported to be around $20-23 \%$ in the general population ${ }^{12,17}$ and much higher (around 52\%) in hypertensive patients. ${ }^{18}$ To our knowledge, there is no study examining the prevalence of MS in persons at high risk for development of T2D, the effect of lifestyle modifications on its frequency, the relationship between impaired glucose metabolism and prevalence of MS and the impact of improving dietary and physical activity habits. The aim of the present study was therefore to evaluate the impact of a one-year, non-intensive, community-based, lifestyle intervention program for prevention of $\mathrm{T} 2 \mathrm{D}$, on the prevalence of the MS in individuals at high risk for developing T2D.

\section{SUBJECTS AND METHODOLOGY}

\section{Study population}

The methods employed in the present study have been described in detail elsewhere..$^{19}$ Briefly, the validated Finnish Type 2 Diabetes Risk Score (FINDRISC) questionnaire ${ }^{20,21}$ was distributed to persons without known diabetes, aged 35-75 years, residing or working in the metropolitan area around Athens, in primary care and occupational settings. This was part of an EU funded project - DE-PLAN ("Diabetes in Europe - Prevention using Lifestyle, Physical Activity and Nutritional Intervention", http://www. ktl.fi/deplan) for the prevention of T2D in the EU member countries. ${ }^{22}$

The distribution procedure resulted in 3,240 completed FINDRISC questionnaires. All highrisk individuals (based on a FINDRISC score $\geq 15$ ) were invited to undergo a standard 75-g oral glucose tolerance test (OGTT) in order to exclude those with unknown diabetes. The remainder were asked to participate in the intervention project. During the baseline OGTT, all individuals were asked to complete a questionnaire regarding their socio-demographic characteristics, as well as nutritional, physical activity and smoking habits, based on the Finnish Diabetes Prevention Study (DPS). ${ }^{23}$ Blood pressure (BP) was measured twice, 2 min apart, in a sitting position, and the average of the two was recorded. Body-weight and height were measured using a scale and a stadiometer to the nearest $0.5 \mathrm{~kg}$ and $0.5 \mathrm{~cm}$, respectively, and body mass index (BMI; $\mathrm{kg} / \mathrm{m}^{2}$ ) was calculated. Waist circumference (WC) was measured at the mid-point between the iliac crest and the costal arch. All of the above procedures were repeated at the conclusion of the intervention phase, when an OGTT was repeated (one-year follow-up). The entire project (screening and intervention phases) was approved by the ethics committee of the cooperating Laiko General Hospital and the Greek National Drug Organization. All participants gave written informed consent according to the general recommendations of the Declaration of Helsinki. ${ }^{24}$ 


\section{Intervention}

The one-year intervention program consisted of six group-based sessions (lasting one hour each), held bimonthly by a registered dietitian in the area of the participants' residence or work. Groups of 6-10 persons were constructed (Figure 1). ${ }^{19}$

The five prevention goals from the DPS study ${ }^{23}$ comprised the core intervention goals of the sessions. General counseling to increase physical activity to at least 30-40 minutes, five times per week, was given, although no formal exercise sessions took place.
Dietary assessment was conducted both at baseline and at the end of the intervention and was based on a semi-quantitative food frequency questionnaire evaluating habitual consumption of the basic food groups, based on the DPS study. ${ }^{23}$ The dietary aims of the intervention were combined to form a scoring system (intervention's dietary scoring system - IDS score) and this was used to evaluate the participants' dietary habits both at baseline and at the end of the intervention..$^{25}$ Briefly, the baseline consumption of the recorded food items (i.e. fruits, vegetables, refined cereals, sugars, whole fat dairy products

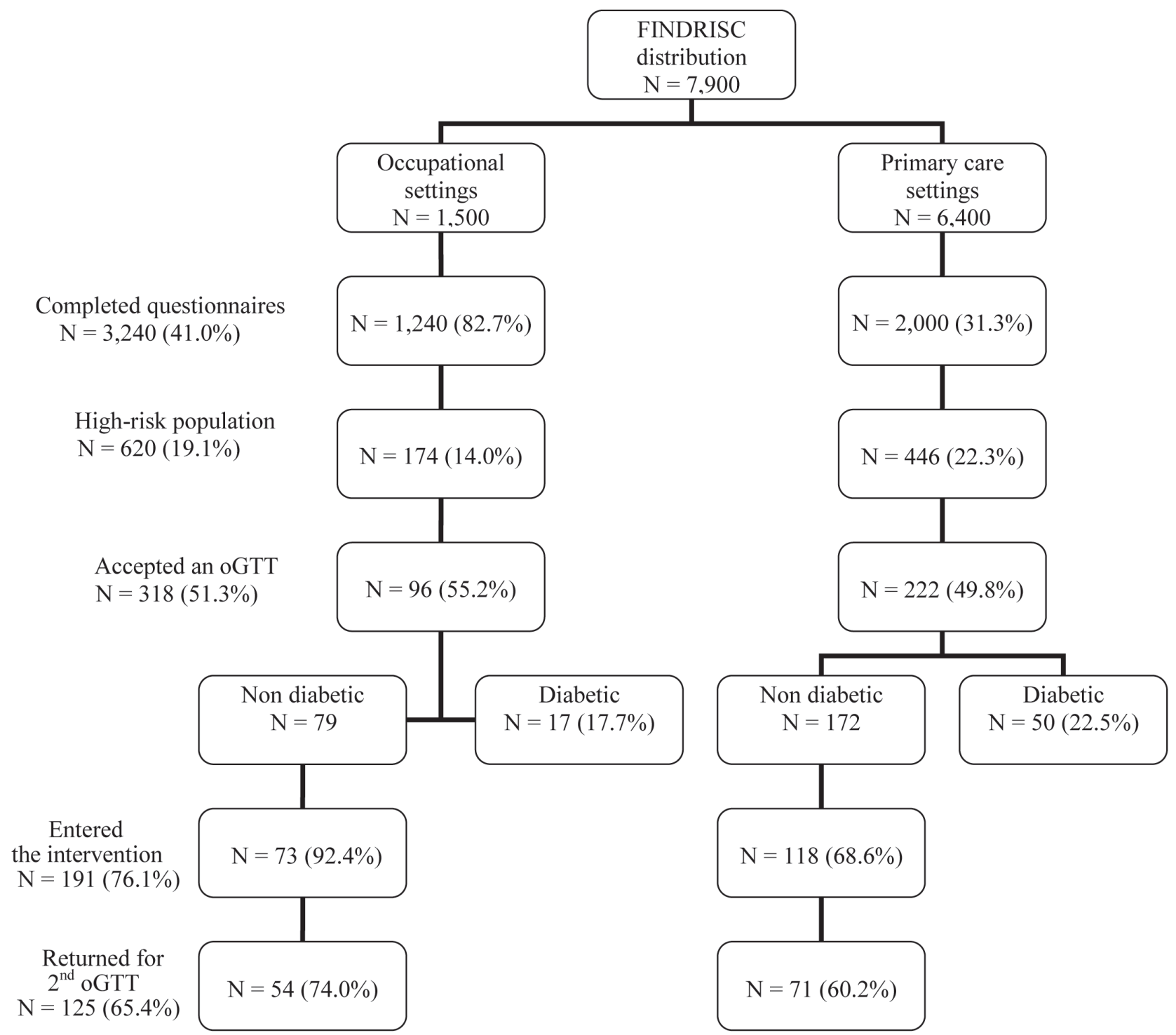

Figure 1. Flowchart of the various phases of the project, with number of FINDRISC questionnaires distribution, recruitment of persons at the occupational and primary-care setting and follow-up interventions. 
and processed meats) was divided into quartiles; for the beneficial foods (fruits and vegetables) the first quartile was assigned a value of 1 and the fourth a value of 4, whereas for the unhealthy foods (refined cereals, sugars, whole fat dairy products and processed meats) the first quartile was assigned a value of 4 and the fourth a value of 1 . Then, the sum of these 6 values (corresponding to each food group) was calculated (IDS score), the final relevant score ranging from 6 to 24 . The same score was calculated at the one-year follow-up visit. An increase in the score was considered to reflect favourable changes, according to the intervention's goals.

\section{Biochemical assessment}

Blood samples were collected after a 12-hour fast, at the baseline and the one-year OGTT. Plasma glucose, total and high-density lipoprotein (HDL) cholesterol and triglyceride levels as well as insulin levels were measured at a central accredited university research laboratory, using enzymatic assays. Lowdensity lipoprotein (LDL) cholesterol was calculated using the Friedewald formula. ${ }^{26}$

\section{Prevalence of $M S$}

The presence of MS was assessed based on the revised AHA/NHLBI definition, ${ }^{8}$ both at baseline and at the one-year follow-up visit, specifically, using three or more of the following conditions: waist circumference $>102 \mathrm{~cm}$ in men ( $>88 \mathrm{~cm}$ in women); serum triglyceride level $\geq 150 \mathrm{mg} / \mathrm{dl}(1.7 \mathrm{mmol} / \mathrm{L})$ or treatment for high triglycerides; HDL-cholesterol level $<40 \mathrm{mg} / \mathrm{dl}(1.03 \mathrm{mmol} / \mathrm{L})$ in men, [ $<50 \mathrm{mg} / \mathrm{dl}$ $(1.30 \mathrm{mmol} / \mathrm{L})$ in women] or treatment for low HDLcholesterol; BP >130/85 mm Hg or treatment with antihypertensive medications; and FPG level $\geq 100$ $\mathrm{mg} / \mathrm{dl}(5.6 \mathrm{mmol} / \mathrm{L})$.

\section{Statistical analysis}

Continuous variables are presented as mean \pm one-standard deviation, while qualitative variables as absolute and relative frequencies (\%). Normality of distributions was evaluated with the KolmogorovSmirnov test. Comparisons between normally distributed continuous variables were performed with the calculation of the paired samples Student's t-test, while non-normally distributed variables were carried out with the Related Samples Wilcoxon Signed
Rank test. Associations between categorical variables were tested with the use of contingency tables and the calculation of the chi-squared test. Pearson's correlation coefficient (or Spearman's correlation coefficient where appropriate) was used to investigate associations between variables. To explore potential independent baseline predictors for improvement of MS status, multiple logistic regression analysis was performed and the likelihood of improving the status was estimated (improvement of MS status was defined as resolution of MS in individuals with baseline presence of the syndrome). All reported p-values are from two-sided tests and a significance level of $5 \%$. Data were analyzed using the Statistical Package SPSS, version 19.0 (SPSS Inc., Chicago, IL, USA).

\section{RESULTS}

Out of the 3,240 completed FINDRISC questionnaires, 620 high-risk individuals were identified and $318(51.3 \%)$ agreed to undergo an OGTT. After excluding $67(21.1 \%)$ persons with screen-detected diabetes, 191 individuals (from the remaining 251 ) - aged $56.3 \pm 10.8$ years ( $40 \%$ males) - agreed to participate in the intervention phase of the project. At the one-year conclusion visit, 125 individuals $(65.4 \%)$ had a full reassessment and are included in the present analysis. The remaining 66 participants either refused the second OGTT or were lost during the follow-up period. They did not differ regarding age, BMI, body-weight, waist circumference, blood pressure and baseline glucose or lipid parameters from those who completed the project (Figure 1).

Table 1 shows anthropometric and clinical data of the participants at baseline and one year after the intervention. They all attended at least one intervention session with the dietitians $(9.7 \%$ attended one session, $4.0 \%$ two sessions, $12.9 \%$ three sessions, $12.1 \%$ four, $26.6 \%$ five and $34.7 \%$ all six sessions) and displayed favourable changes in their glycemic status as well as their dietary and lipid parameters ${ }^{19,25}$. The prevalence of the MS at baseline was $63.4 \pm 48.4 \%$ and decreased to $54.8 \pm 50.0 \%$ at the end of the intervention $(\mathrm{p}<0.001)$. Out of the five components of the MS, only systolic BP demonstrated a significant decrease after the intervention (129.8 \pm 15.8 at baseline vs. $126.7 \pm 18.0 \mathrm{mmHg}$ one year later, $\mathrm{p}=0.029)$. 
Table 1. Anthropometric and clinical data of the participants at baseline and one year after the intervention [mean (SD)], unless otherwise stated) $(\mathrm{n}=125)$

\begin{tabular}{|c|c|c|c|c|}
\hline Characteristic & Baseline & 1 yr after & Difference (Baseline-1 year after) & $\mathbf{P}$ \\
\hline Weight (kg) & $89.1(13.4)$ & $88.1(13.6)$ & $1.0(4.8)$ & 0.025 \\
\hline BMI $\left(\mathrm{kg} / \mathrm{m}^{2}\right)$ & $32.1(4.2)$ & $31.6(4.0)$ & $0.5(2.1)$ & 0.015 \\
\hline Waist circumference (cm) & $103.0(11.0)$ & $102.7(10.6)$ & $0.3(6.8)$ & 0.599 \\
\hline \multicolumn{5}{|l|}{$\mathrm{BP}(\mathrm{mmHg})$} \\
\hline Systolic & $129.8(15.8)$ & $126.7(18.0)$ & $3.1(15.8)$ & 0.029 \\
\hline Diastolic & $78.8(11.4)$ & $79.9(10.5)$ & $-1.1(13.6)$ & 0.361 \\
\hline Glucose fasting $(\mathrm{mg} / \mathrm{dL})$ & $104.7(11.3)$ & $103.9(24.1)$ & $0.8(24.2)$ & 0.699 \\
\hline Glucose 2 hrs (mg/dL) & $118.4(32.3)$ & $122.2(52.2)$ & $-3.8(48.3)$ & 0.384 \\
\hline Total cholesterol (mg/dL) & $228.1(34.1)$ & $214(36.9)$ & $14.1(38.2)$ & $<0.001$ \\
\hline Triglycerides (mg/dL) (median) & 112.0 & 109.5 & -2.5 & 0.857 \\
\hline HDL-C (mg/dL) & $49.8(8.5)$ & $49.8(8.2)$ & $0.0(2.9)$ & 0.925 \\
\hline LDL-C (mg/dL) & $154.2(30.5)$ & $139.4(35.4)$ & $14.8(35.1)$ & $<0.001$ \\
\hline IDS & $15.8(2.9)$ & $16.6(3.1)$ & $-0.8(3.0)$ & 0.003 \\
\hline Exercise at work and leisure (min/day) & $37.2(28.3)$ & $34.1(25.8)$ & $3.1(31.4)$ & 0.325 \\
\hline $\operatorname{MS}(\%)$ & $63.4(48.4)$ & $54.8(50.0)$ & $8.6(55.4)$ & $<0.001$ \\
\hline
\end{tabular}

BMI: Body Mass Index; BP: Blood Pressure; HDL: High Density Lipoprotein; LDL: Low Density Lipoprotein; IDS: Intervention's Dietary Score; MS: Metabolic syndrome.

Table 2 shows the prevalence of MS and each of its components at baseline, by age group and by gender. The prevalence of MS was similar across all age groups and the two genders. However, significant differences by age group emerged for the prevalence of high BP (increasing with age, $p<0.001$ ) and for the prevalence of high waist circumference and low HDL-cholesterol for the two genders (both higher in women). Of note, high waist circumference was the commonest component of all (82\% of participants). The rest of MS components were similar across all age groups and the two genders. The severity of MS, in terms of the number of positive criteria (mean, for the whole group: 2.8), did not differ by age group or gender. The majority (50 persons [40.7\%]) had three positive criteria, 24 participants $(19.5 \%)$ had four and

Table 2. Prevalence of MS and its components by age and gender at baseline

\begin{tabular}{|c|c|c|c|c|c|c|c|}
\hline \multirow[b]{2}{*}{ Variable } & \multirow{2}{*}{$\begin{array}{c}\text { All } \\
\text { participants } \\
(n=125)\end{array}$} & \multicolumn{4}{|c|}{ Age groups } & \multicolumn{2}{|c|}{ Gender } \\
\hline & & $\begin{array}{c}<45 y \\
(n=21)\end{array}$ & $\begin{array}{l}45-54 y \\
(n=46)\end{array}$ & $\begin{array}{l}55-64 y \\
(n=33)\end{array}$ & $\begin{array}{c}>64 y \\
(n=24)\end{array}$ & $\begin{array}{c}\text { Men } \\
(n=55)\end{array}$ & $\begin{array}{l}\text { Women } \\
(n=68)\end{array}$ \\
\hline Metabolic syndrome, n (\%) & $78(63)$ & $11(52)$ & $29(64)$ & $23(72)$ & $15(63)$ & $35(64)$ & $43(63)$ \\
\hline High WC, n $(\%)^{\#}$ & $102(82)$ & $16(76)$ & $38(83)$ & $30(91)$ & $18(75)$ & $42(74)$ & $60(88)$ \\
\hline Low HDL-C or receiving treatment, n $(\%)^{@}$ & $34(28)$ & $8(38)$ & $15(33)$ & $6(19)$ & $5(21)$ & $5(9)$ & $29(43)$ \\
\hline High Trigl or receiving treatment, $\mathrm{n}(\%)$ & $32(26)$ & $2(10)$ & $15(33)$ & $10(31)$ & $4(17)$ & $17(31)$ & $15(22)$ \\
\hline High FPG, n $(\%)$ & $84(67)$ & $13(62)$ & $30(65)$ & $23(70)$ & $38(75)$ & $43(75)$ & $41(60)$ \\
\hline High $\mathrm{BP}$ or receiving treatment, $\mathrm{n}(\%)^{+}$ & $93(76)$ & $13(62)$ & $31(67)$ & $28(85)$ & $21(91)$ & $44(79)$ & $49(73)$ \\
\hline
\end{tabular}

WC: Waist circumference; HDL-C: High Density Lipoprotein cholesterol; Trigl: Triglycerides; FPG: Fasting plasma glucose; BP: Blood Pressure ${ }^{\#} \mathrm{p}<0.05$ for the difference between genders, ${ }^{\circledR} \mathrm{p}<0.001$ for the difference between genders, ${ }^{+} \mathrm{p}<0.05$ for the differences across age groups (by one-way ANOVA). 
only four persons (3.3\%) fulfilled all five criteria.

Table 3 shows the incidence of MS and its components among participants who did not have them at baseline, as well as the resolution of MS and its components among those who had them at baseline. In particular, among participants who did not have MS at baseline [44 persons (36\%)], a total of 14 individuals $(31.8 \%)$ developed the syndrome at the end of the intervention period one year later, and among participants who had MS at baseline (78 persons [63\%]) it was resolved in 24 individuals $(31 \%)$ one year later. Eighty-four participants $(68 \%)$ remained in the same status at the end of the intervention. When the 78 persons who had MS at baseline were analyzed separately, improvement in MS status after one year was significantly associated with male gen$\operatorname{der}(\mathrm{r}=0.24, \mathrm{p}=0.037)$, having a lower dietary score at baseline $(\mathrm{r}=-0.25, \mathrm{p}=0.028)$, improvement of the dietary score after one year $(r=0.24, p=0.033)$, having a higher baseline HDL-C level $(r=0.23, p=0.040)$, a lower baseline triglyceride level $(\mathrm{r}=-0.23, \mathrm{p}=$ $0.048)$, a lower FINDRISC score $(r=-0.30, p=0.007)$ and having a lower number of MS components at baseline $(r=-0.32, p=0.004)$.

In a multiple logistic regression model with backward selection of variables, including the variables

Table 3. Incidence or resolution of MS and its components at one year

\begin{tabular}{lcc}
\hline & $\begin{array}{c}\text { Incidence among } \\
\text { participants not } \\
\text { meeting criterion } \\
\text { at baseline }\end{array}$ & $\begin{array}{c}\text { Resolution } \\
\text { among } \\
\text { participants } \\
\text { and its compoting criterion } \\
\text { at baseline }\end{array}$ \\
\hline $\begin{array}{l}\text { Metabolic syndrome, } \\
\mathrm{n}(\%)\end{array}$ & $14(32)$ & $24(31)$ \\
$\begin{array}{l}\text { High WC, n (\%) } \\
\text { Low HDL-C or } \\
\text { receiving treatment, } \\
\mathrm{n}(\%)\end{array}$ & $9(39)$ & $11(11)$ \\
$\begin{array}{l}\text { High Trigl or receiving } \\
\text { treatment, n }(\%)\end{array}$ & $3(3)$ & $5(15)$ \\
$\begin{array}{l}\text { High FPG, n }(\%) \\
\text { High BP or receiving } \\
\text { treatment, n }(\%)\end{array}$ & $14(16)$ & $11(34)$ \\
\hline
\end{tabular}

WC: Waist circumference; HDL-C: High Density Lipoprotein cholesterol; Trigl: Triglycerides; FPG: Fasting plasma glucose; BP: Blood Pressure. that showed significant correlations in the univariate analyses, younger age $(\beta=-0.16, p=0.009)$, male gen$\operatorname{der}(\beta=4.44, p=0.004)$, improvement of the dietary score after one year $(\beta=3.34, p=0.022)$, a lower FINDRISC score $(\beta=-0.67, p=0.033)$, a lower triglyceride level $(\beta=-10.12, p=0.010)$ and a higher HDL-C level $(\beta=0.32, p=0.003)$ were significantly and independently associated with improvement in MS status.

\section{DISCUSSION}

The data in the present study show that the prevalence of MS in middle-aged persons at high risk for the development of T2D is quite high $(63.4 \pm 48.4 \%)$ and that a one-year, non-intensive lifestyle intervention program is able to decrease it significantly, possibly conferring multiple benefits regarding CVD risk. These data confirm the findings of the U.S. Diabetes Prevention Program which was performed in a much larger and more ethnically diverse population of individuals with IFG/IGT ${ }^{13}$ where, apart from diabetes prevention, the additional possibility of cardiovascular prevention was also entertained through decrease of MS prevalence with lifestyle intervention after three years of follow-up. The prevalence of MS in the DPP population was nearly the same as in the present study $(69 \%$, when an FPG cut-off $>100 \mathrm{mg} / \mathrm{dl}[5.6$ $\mathrm{mmol} / \mathrm{L}]$ was used) and, similar to our findings, it was not different among age groups studied. On the contrary, in the general population the prevalence increases with age (from $12 \%$ for individuals in their thirties, up to around $42 \%$ at age $>70$ years). ${ }^{10}$ This can be explained by the fact that our population, as expected, had a higher cardiovascular and diabetes risk, since the participants were specifically selected (by the FINDRISC questionnaire) to be at increased risk for T2D.

Of particular note is the distribution of components of the syndrome: increased waist circumference (82\%), high BP (76\%) and high FPG (67\%) were the most prevalent components (Table 2). In the DPP data set, WC was also the most prevalent component (78\%), but low HDL-C (57\%) and high triglyceride levels $(46 \%)$ were the next more frequent ones. A difference in the definition of FPG (>110 mg/dl [6.1 $\mathrm{mmol} / \mathrm{L}]$ and ethnic variability in the DPP study) and 
a smaller number of participants in our study could possibly explain these differences.

A novel finding of the present study was the identification of the factors that contributed to the improvement of MS status in those affected at baseline: improvement in the dietary score after one year of participation in the program was identified as a major contributor, which highlights the importance of diet in resolution of MS. Although physical activity has been identified as an important factor in preventing or treating $\mathrm{MS},{ }^{27}$ it was not an important contributor in the present study. Time spent on exercise though remained unchanged during the intervention ${ }^{25}$ and this could be partly attributed to the fact that the intervention goal was not very intense and no session was exclusively devoted to exercise. The fact that males showed a better resolution of MS than females was not observed in the DPP study and, if confirmed in other studies, could be a message to healthcare providers and policymakers for more stringent and intense focus on women as a target population for prevention of MS and CVD risk in general.

To our knowledge, with the exception of the DPP, ${ }^{13}$ other randomized controlled trials with the prevention or treatment of MS as the main outcome have not been published. Several large randomized controlled trials provide strong evidence that favourable lifestyle changes, including diet and regular physical activity, are effective in the prevention of T2D in individuals who are overweight and have impaired glucose tolerance. ${ }^{23,28,29}$ The present study showed that apart from improvement in the glucose status, ${ }^{19}$ other CVD risk factors that constitute components of MS were also improved with the implementation of a non-intensive lifestyle modification program, which confers a substantial CVD risk benefit. In particular, increased WC was improved in $11 \%$ of the participants, low HDL-C levels in $15 \%$, high triglycerides in $34 \%$, high FPG in $37 \%$ and high BP in $12 \%$ (Table 3 ). In comparison, in the DPP data set, ${ }^{13}$ the respective changes in the intensive lifestyle intervention group were for WC $29 \%$, for low HDL-C $22 \%$, for high triglycerides $40 \%$, for high FPG $45 \%$ and for high BP 32\%. A more intensive lifestyle modification program in the DPP study, where each participant had 16 individual sessions - covering diet, exercise and behavioral changes - with a case manager in the first six months of the study and monthly thereafter, could be the explanation for the better results. ${ }^{30}$

The major limitation of our study was the small number of participants (78 persons fulfilled MS definition at baseline) and the relatively short duration of the follow-up (one year). Despite that, most of our findings are consistent with the much larger and longer DPP study. Another limitation is the lack of a control group. However, this is due to the fact that the primary aim of the entire European DEPLAN study $^{22}$ was to test the feasibility of implementing a lifestyle intervention program to prevent $\mathrm{T} 2 \mathrm{D}$ within the national healthcare system of each participating country and not to test the effectiveness of a lifestyle intervention relative to placebo per se, which has already been proven in the previous lifestyle intervention studies. ${ }^{23,28,29}$

One strength of our study was that OGTTs were performed on all participants, both at baseline and at one year after the intervention and thus diabetes incidence was much more reliably discerned than via reliance only on FPG values. In addition, the anthropometric variables (weight, height and waist circumference) were professionally measured and not self-reported. Furthermore, all 191 participants attended several intervention sessions (satisfactory compliance). Of note also is the fact that participants in our study were not all exclusively in the IFG/IGT status, since they were identified earlier through a diabetes risk-score questionnaire. Hence, not only subjects with dysglycemia at baseline, but also with normal glucose tolerance (albeit still at high risk for T2D) were included. Thus, our findings are probably more significant compared to those of other studies that included exclusively IFG/IGT individuals. ${ }^{13}$ In conclusion, our results show that a one-year, nonintensive lifestyle intervention program to prevent T2D is effective in decreasing the prevalence of MS in a substantial proportion of high-risk individuals.

\section{ACKNOWLEDGEMENTS}

We would like to thank the following Health Centers/individuals for helping implement the present study: the medical staff of the Health Center of Alimos (especially Dr. Ourania Zacharopoulou), the staff of the Agioi Anargyroi Center for the Elderly, 
the medical and nursing staff of the Health Center of Markopoulo (especially Mrs. R. Salonikioti), the medical and nursing staff of the Hellenic Telecommunications Company (especially Drs C. Pietris, C. Alexopoulos), the medical and nursing staff of the Hellenic Radiotelevision (especially Dr. M. Katsorida), the medical and nursing staff of the Bank of Greece (especially Drs V. Spandagos, P. Konstantopoulou), the medical staff of the Olympic Village complex (especially Dr. S. Tigas), the staff of the electrical equipment manufacturer "Pitsos-Bosch", the medical and nursing staff of the Health Center of Vari (especially Dr. M. Dandoulakis), Dr. K. Kyriakopoulos and Ms. K. Perrea.

\section{Funding}

This project was funded by the Commission of the European Communities, Directorate C - Public Health, grant agreement No. 2004310. Under the rules of the agreement, it was also co-funded by the private sector and in this case it was supported by an unrestricted educational grant from Bristol-MyersSquibb, Greece.

\section{Disclosure statement:}

Nothing to declare

\section{REFERENCES}

1. Reaven GM, 1988 Banting lecture 1988 Role of insulin resistance in human disease. Diabetes 37: 1595-1607.

2. Gami AS, Witt BJ, Howard DE, et al, 2007 Metabolic syndrome and risk of incident cardiovascular events and death: a systematic review and meta-analysis of longitudinal studies. J Am Coll Cardiol 49: 403-414.

3. Ford ES, Li C, Sattar N, 2008 Metabolic syndrome and incident diabetes: current state of the evidence. Diabetes Care 31: 1898-1904.

4. Alberti KG, Zimmet PZ, 1998 Definition, diagnosis and classification of diabetes mellitus and its complications. Part 1: diagnosis and classification of diabetes mellitus provisional report of a WHO consultation. Diabet Med 15: 539-553.

5. Balkau B, Charles MA, 1999 Comment on the provisional report from the WHO consultation. European Group for the Study of Insulin Resistance (EGIR). Diabet Med 16: 442-443.

6. Executive summary of the third report of the National Cholesterol Education Program (NCEP), 2001 Expert panel on detection, evaluation, and treatment of high blood cholesterol in adults (Adult Treatment Panel III). JAMA 285: 2486-2497.
7. Alberti KG, Zimmet P, Shaw J, 2005 The metabolic syndrome-a new worldwide definition. Lancet 366: 1059-1062

8. Grundy SM, Cleeman JI, Daniels SR, et al, 2005 Diagnosis and management of the metabolic syndrome: an American Heart Association/National Heart, Lung, and Blood Institute Scientific Statement. Circulation 112: 2735-2752.

9. Alberti KG, Eckel RH, Grundy SM, et al, 2009 Harmonizing the metabolic syndrome: a joint interim statement of the International Diabetes Federation Task Force on Epidemiology and Prevention; National Heart, Lung, and Blood Institute; American Heart Association; World Heart Federation; International Atherosclerosis Society; and International Association for the Study of Obesity. Circulation 120: 1640-1645.

10. Park YW, Zhu S, Palaniappan L, Heshka S, Carnethon MR, Heymsfield SB, 2003 The metabolic syndrome: prevalence and associated risk factor findings in the US population from the Third National Health and Nutrition Examination Survey, 1988-1994. Arch Intern Med 163: 427-436.

11. Doupis J, Dimosthenopoulos C, Diamanti K, Perrea D, Katsilambros N, Makrilakis K, 2009 Metabolic syndrome and Mediterranean dietary pattern in a sample of young, male, Greek navy recruits. Nutr Metab Cardiovasc Dis 19: e 7-8.

12. Panagiotakos DB, Pitsavos C, Chrysohoou C, et al, 2004 Impact of lifestyle habits on the prevalence of the metabolic syndrome among Greek adults from the ATTICA study. Am Heart J 147: 106-112.

13. Orchard TJ, Temprosa M, Goldberg R, et al, 2005 The effect of metformin and intensive lifestyle intervention on the metabolic syndrome: the Diabetes Prevention Program randomized trial. Ann Intern Med 142: 611-619.

14. Ford ES, Giles WH, Dietz WH, 2002 Prevalence of the metabolic syndrome among US adults: findings from the third National Health and Nutrition Examination Survey. JAMA 287: 356-359.

15. Ford ES, Giles WH, Mokdad AH, 2004 Increasing prevalence of the metabolic syndrome among U.S. adults. Diabetes Care 27: 2444-2449.

16. Ford ES, 2005 Prevalence of the metabolic syndrome defined by the International Diabetes Federation among adults in the U.S. Diabetes Care 28: 2745-2749.

17. Athyros VG, Bouloukos VI, Pehlivanidis AN, Papageorgiou AA, Dionysopoulou SG, Symeonidis AN, et al, 2005 The prevalence of the metabolic syndrome in Greece: the MetS-Greece Multicentre Study. Diabetes Obes Metab 7: 397-405.

18. Lioudaki E, Vrentzos GE, Mavrogeni H, et al, 2012 Prevalence of Metabolic Syndrome According to Different Definitions in a Hypertensive Population. Angiology 63: $39-47$

19. Makrilakis K, Liatis S, Grammatikou S, Perrea D, Katsilambros N, 2010 Implementation and effectiveness of 
the first community lifestyle intervention programme to prevent Type 2 diabetes in Greece. The DE-PLAN study. Diabet Med 27: 459-465.

20. Saaristo T, Peltonen M, Lindstrom J, et al, 2005 Crosssectional evaluation of the Finnish Diabetes Risk Score: a tool to identify undetected type 2 diabetes, abnormal glucose tolerance and metabolic syndrome. Diab Vasc Dis Res 2: 67-72.

21. Makrilakis K, Liatis S, Grammatikou S, et al, 2011 Validation of the Finnish Diabetes Risk Score Questionnaire (FINDRISC) to Screen for Undiagnosed Type 2 Diabetes, Dysglycaemia and the Metabolic Syndrome in Greece. Diabetes Metab 32: 144-151.

22. Schwartz PEH, Lindstrom J, Kissimova-Skarbeck K, et al, on behalf of the DE-PLAN project, 2008 The European perspective of Type 2 diabetes prevention: Diabetes in Europe - Prevention using lifestyle, physical activity and nutritional intervention (DE-PLAN) project. Exp Clin Endocrinol Diabetes 116: 167-172.

23. Tuomilehto J, Lindstrom J, Eriksson JG, Valle TT, Hamalainen H, Ilanne-Parikka P, et al, 2001 Prevention of type 2 diabetes mellitus by changes in lifestyle among subjects with impaired glucose tolerance. N Engl J Med 344: 1343-1350.

24. World Medical Association declaration of Helsinki 1997
Recommendations guiding physicians in biomedical research involving human subjects. JAMA 277: 925-926.

25. Kontogianni M, Liatis S, Grammatikou S, Perrea D, Katsilambros N, Makrilakis K, 2012 Changes in dietary habits and their association with metabolic markers after a non-intensive, community-based lifestyle intervention to prevent type 2 diabetes, in Greece. The DEPLAN study. Diabetes Res Clin Pract 95: 207-214.

26. Friedewald WT, Levy RI, Fredrickson DS, 1972 Estimation of the concentration of low density lipoprotein cholesterol in plasma, without use of the preparative ultracentifuge. Clin Chem 18: 499-502.

27. Lakka TA, Laaksonen DE, 2007 Physical activity in prevention and treatment of the metabolic syndrome. Appl Physiol Nutr Metab 32: 76-88.

28. Pan XR, Li GW, Hu YH, et al, 1997 Effects of diet and exercise in preventing NIDDM in people with impaired glucose tolerance. The Da Qing IGT and Diabetes Study. Diabetes Care 20: 537-544.

29. Knowler WC, Barrett-Connor E, Fowler SE, et al, 2002 Reduction in the incidence of type 2 diabetes with lifestyle intervention or metformin. N Engl J Med 346: 393-403.

30. The Diabetes Prevention Program, 1999 Design and methods for a clinical trial in the prevention of type 2 diabetes. Diabetes Care 22: 623-634. 